\title{
Effet des paramètres de conception du piston sur le frottement jupe-chemise
}

\author{
Ridha Mazouzi ${ }^{1, a}$, Patrick Maspeyrot ${ }^{1}$, Ahmed Kellaci ${ }^{2}$ et Driss Djaouad Rahal ${ }^{3}$ \\ 1 Laboratoire de Mécanique des solides, Université de Poitiers, UMR CNRS 6610, Bd Pierre et Marie Curie, BP 30179, \\ 86962 Futuroscope Chasseneuil Cedex, France \\ 2 Centre Universitaire de Khemis-Miliana, route de Theniet el-had, 44225 W.Ain-Defla, Algérie \\ 3 Université des Sciences et de la Technologie Mohamed Boudiaf-Oran, BP 1505 El M'naouar, 31000 Oran, Algérie
}

Reçu le 17 octobre 2008, accepté le 18 février 2009

\begin{abstract}
Résumé - Cet article présente un modèle de lubrification mixte en conjonction avec une analyse du mouvement secondaire du piston. Certains paramètres influent ce genre de mouvement tel que l'élasticité de la jupe et la forme de son profil (plat ou bombé). Les résultats montrent que ces paramètres ont une grande influence sur les forces de frottement, et par conséquent, ils ont le potentiel pour optimiser la perte de puissance par frottement du piston.
\end{abstract}

Mots clés : Lubrification / jupe de piston / frottement / puissance dissipée / profil de jupe

\begin{abstract}
Effects of piston design parameters on skirt-liner friction. A mixed lubrication model is presented in this paper. It is developed for use in conjunction with a piston secondary motion analysis. Some parameters affect this kind of movement as the elasticity of the skirt and its profile shape (flat or curved). The results show that these parameters have a great influence on the friction forces, and therefore, they have the potential to optimize the piston friction power loss.
\end{abstract}

Key words: Lubrication / piston skirt / friction / power loss / skirt profile

\section{Introduction}

La jupe du piston dans un moteur à combustion interne joue un rôle important dans la transmission de puissance. Les détails de l'interaction jupe-chemise dépendent de divers paramètres de conception du piston. Dans le but de déterminer l'influence de ces paramètres, d'intenses recherches, à la fois expérimentales et numériques ont été menées sur la lubrification de la jupe et le mouvement secondaire du piston.

La simulation numérique du mouvement secondaire du piston a commencé il y a des décennies [1-5]. Un groupe de chercheurs a développé un modèle numérique du mouvement secondaire du piston avec une lubrification hydrodynamique pour des jupes rigides [6-8]. Certains chercheurs ont déterminé les déformations de la jupe mais sans aucune analyse de la lubrification [9,10]. Depuis lors, des modèles numériques variés sont développés $[1-4,11,12]$,

\footnotetext{
a Auteur pour correspondance : mazouzi_r@yahoo.fr
}

en incluant dans quelques cas le rôle de la lubrification et de l'élasticité de la jupe.

L'assemblage du piston est depuis longtemps reconnu comme la source majeure des frottements mécaniques dans un moteur. C'est la partie où l'on peut réaliser une réduction significative des frottements par des modifications de conception. Les premières mesures de frottement dans un moteur à combustion, par Forbes et Taylor [13] date de 1943. Furuhama et ses associés [14-16], en utilisant un montage flottant de la chemise, ont mesuré les frottements instantanés d'un assemblage de piston sous des conditions de combustion.

Jang et Cho [17] s'intéressent aux profils de la jupe. Pour cela, ils ont étudié le mouvement du piston par la modification des profils de la jupe et le désaxage de l'axe du piston. Ils constatent qu'un profil courbé et un grand désaxage du côté de la poussée fournira de meilleures performances, avec un impact latéral faible durant le cycle moteur. En 2005, Balakrishnan et Rahnejat [18] traitent avec une analyse tribodynamique transitoire le contact 
Nomenclature

\begin{tabular}{|c|}
\hline$a:$ Distance haut jupe-axe du piston (m) \\
\hline$b:$ Distance haut jupe-centre de gravité $(\mathrm{m})$ \\
\hline$C_{i j}$ : Matrice de compliance relative à la pression $\left(\mathrm{m} \cdot \mathrm{Pa}^{-1}\right)$ \\
\hline$C_{\mathrm{g}}:$ Désaxage centre de gravité du piston $(\mathrm{m})$ \\
\hline$C_{\mathrm{p}}:$ Désaxage axe du piston $(\mathrm{m})$ \\
\hline$E^{\prime}$ : Module de Young de la jupe (GPa) \\
\hline$e_{\mathrm{h}}, e_{\mathrm{b}}:$ Excentricités du piston (haut, bas) $(\mathrm{m})$ \\
\hline$F:$ Force normale agissant sur les côtés de la jupe $(\mathrm{N})$ \\
\hline$F_{\mathrm{b}}:$ Force de la bielle $(\mathrm{N})$ \\
\hline$F_{\mathrm{c}}:$ Force de contact $(\mathrm{N})$ \\
\hline$F_{\text {gaz }}:$ Force des gaz de combustion $(\mathrm{N})$ \\
\hline$F_{\mathrm{f}}:$ Force de frottement totale $(\mathrm{N})$ \\
\hline$F_{\mathrm{fc}}:$ Force de frottement de contact $(\mathrm{N})$ \\
\hline$F_{\text {fh }}:$ Force de frottement hydrodynamique $(\mathrm{N})$ \\
\hline$F_{\mathrm{h}}:$ Force hydrodynamique $(\mathrm{N})$ \\
\hline$F_{i \mathrm{a}_{\mathrm{a}} x}, F_{i \mathrm{a} \_y}:$ Forces d'inertie dues à la masse de l'axe $(\mathrm{N})$ \\
\hline$F_{i \mathrm{p} \_x}, F_{i_{\mathrm{p} \_} y}:$ Forces d'inertie dues à la masse du piston $(\mathrm{N})$ \\
\hline$h:$ Épaisseur du film lubrifiant $(\mathrm{m})$ \\
\hline$h_{\mathrm{e}}:$ Déformation élastique de la jupe $(\mathrm{m})$ \\
\hline$I_{\mathrm{p}}:$ Inertie de rotation du piston $\left(\mathrm{kg} \cdot \mathrm{m}^{2}\right)$ \\
\hline$L:$ Longueur de la jupe du piston $(\mathrm{m})$ \\
\hline$L_{\mathrm{w}}:$ Longueur de l'ondulation $(\mathrm{m})$ \\
\hline$l:$ Longueur de la bielle $(\mathrm{m})$ \\
\hline$M$ : Moment total dû aux forces normales (N.m) \\
\hline$M_{\mathrm{c}}:$ Moment de la force de contact (N.m) \\
\hline$M_{\mathrm{f}}:$ Moment des forces de frottement (N.m) \\
\hline$M_{\mathrm{fc}}:$ Moment des forces de frottement de contact (N.m) \\
\hline $\begin{aligned} M_{\mathrm{fh}}: & \text { Moment des forces de frottement } \\
& \text { hydrodynamique (N.m) }\end{aligned}$ \\
\hline$M_{\mathrm{h}}$ : Moment des forces hydrodynamiques (N.m) \\
\hline
\end{tabular}

$a$ : Distance haut jupe-axe du piston $(\mathrm{m})$

$b$ : Distance haut jupe-centre de gravité $(\mathrm{m})$

$C_{i j}$ : Matrice de compliance relative à la pression $\left(\mathrm{m} . \mathrm{Pa}^{-1}\right)$

$C_{\mathrm{g}}$ : Désaxage centre de gravité du piston $(\mathrm{m})$

$C_{\mathrm{p}}$ : Désaxage axe du piston $(\mathrm{m})$

$E^{\prime}$ : Module de Young de la jupe $(\mathrm{GPa})$

$e_{\mathrm{h}}, e_{\mathrm{b}}$ : Excentricités du piston (haut, bas) (m)

$F_{\mathrm{b}}:$ Force de la bielle $(\mathrm{N})$

$F_{\mathrm{c}}:$ Force de contact $(\mathrm{N})$

$F_{\text {gaz }}$ : Force des gaz de combustion $(\mathrm{N})$

$F_{\mathrm{f}}:$ Force de frottement totale $(\mathrm{N})$

$F_{\mathrm{fc}}:$ Force de frottement de contact $(\mathrm{N})$

$F_{\text {fh }}$ : Force de frottement hydrodynamique $(\mathrm{N})$

$F_{\mathrm{h}}$ : Force hydrodynamique $(\mathrm{N})$

$F_{i \mathrm{a}_{-} x}, F_{i \mathrm{a}_{-} y}$ : Forces d'inertie dues à la masse de l'axe $(\mathrm{N})$

$F_{i \mathrm{p} \_x}, F_{i \mathrm{p} \_y}:$ Forces d'inertie dues à la masse du piston $(\mathrm{N})$

$h$ : Épaisseur du film lubrifiant $(\mathrm{m})$

$h_{\mathrm{e}}$ : Déformation élastique de la jupe $(\mathrm{m})$

$I_{\mathrm{p}}$ : Inertie de rotation du piston $\left(\mathrm{kg} \cdot \mathrm{m}^{2}\right)$

$L$ : Longueur de la jupe du piston $(\mathrm{m})$

:Longueur de l'ondulation $(\mathrm{m})$

$M$ : Moment total dû aux forces normales (N.m)

$M_{\mathrm{c}}$ : Moment de la force de contact (N.m)

$M_{\mathrm{f}}$ : Moment des forces de frottement (N.m)

$M_{\mathrm{fc}}$ : Moment des forces de frottement de contact (N.m)

$M_{\mathrm{h}}$ : Moment des forces hydrodynamiques (N.m)

de la jupe du piston avec la paroi du cylindre sous des conditions isothermes, en intégrant des profils réalistes de la jupe du piston.

Mansouri et Wong [19] publient en 2005 une étude sur la rugosité des surfaces et la topographie de la jupe à la fois dans la direction axiale et circonférentielle. Trois modes de lubrification (hydrodynamique, mixte et un régime de lubrification limite) sont considérés. La déformation de la jupe est un facteur essentiel du modèle. Ce modèle incorpore les effets des ondulations, de la rugosité de la surface de la jupe du piston, le profil axial de la jupe, la déformation élastique et thermique, la lubrification et le frottement. Ces auteurs ont conclu que des paramètres tels que les ondulations de surface, l'épaisseur du film d'huile, le profil et la flexibilité de la jupe ont un effet majeur sur le frottement de la jupe du piston.

En 2007, Livanos et Kyrtatos [20] présentent un modèle de frottement de l'assemblage du piston (segments, jupe et axe de piston). L'effet de la vitesse du moteur et de la charge sur le frottement a été examiné et comparé avec les résultats d'autres modèles

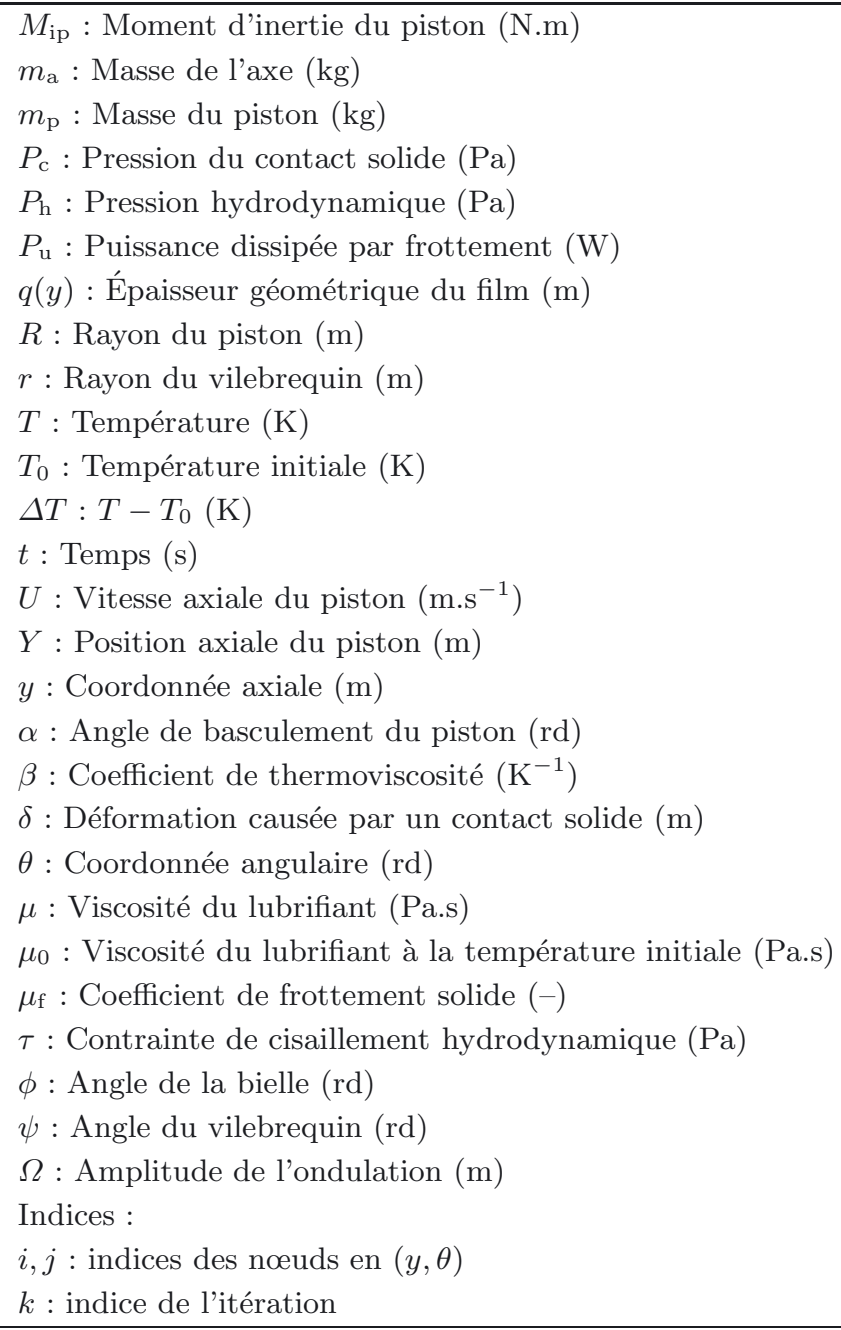

semi-empiriques. Leur modèle permet la simulation détaillée des conditions de lubrification qui prévalent sur l'assemblage du piston.

Dans cet article, un modèle mathématique d'une jupe de piston en lubrification mixte est présenté. Il considère les effets du profil de la surface axiale de la jupe du piston et les déformations élastiques sur la force de frottement et la puissance dissipée. Le champ de pression hydrodynamique est calculé par la méthode des différences finies, un modèle de contact des aspérités est utilisé pour le calcul des pressions de contact, la méthode des éléments-finis est utilisée pour le calcul des déformations des solides.

\section{2 Équations de base}

\section{1 Équation de mouvement}

Le mouvement du piston est décrit par les lois traditionnelles de Newton appliquées au mouvement vertical, 

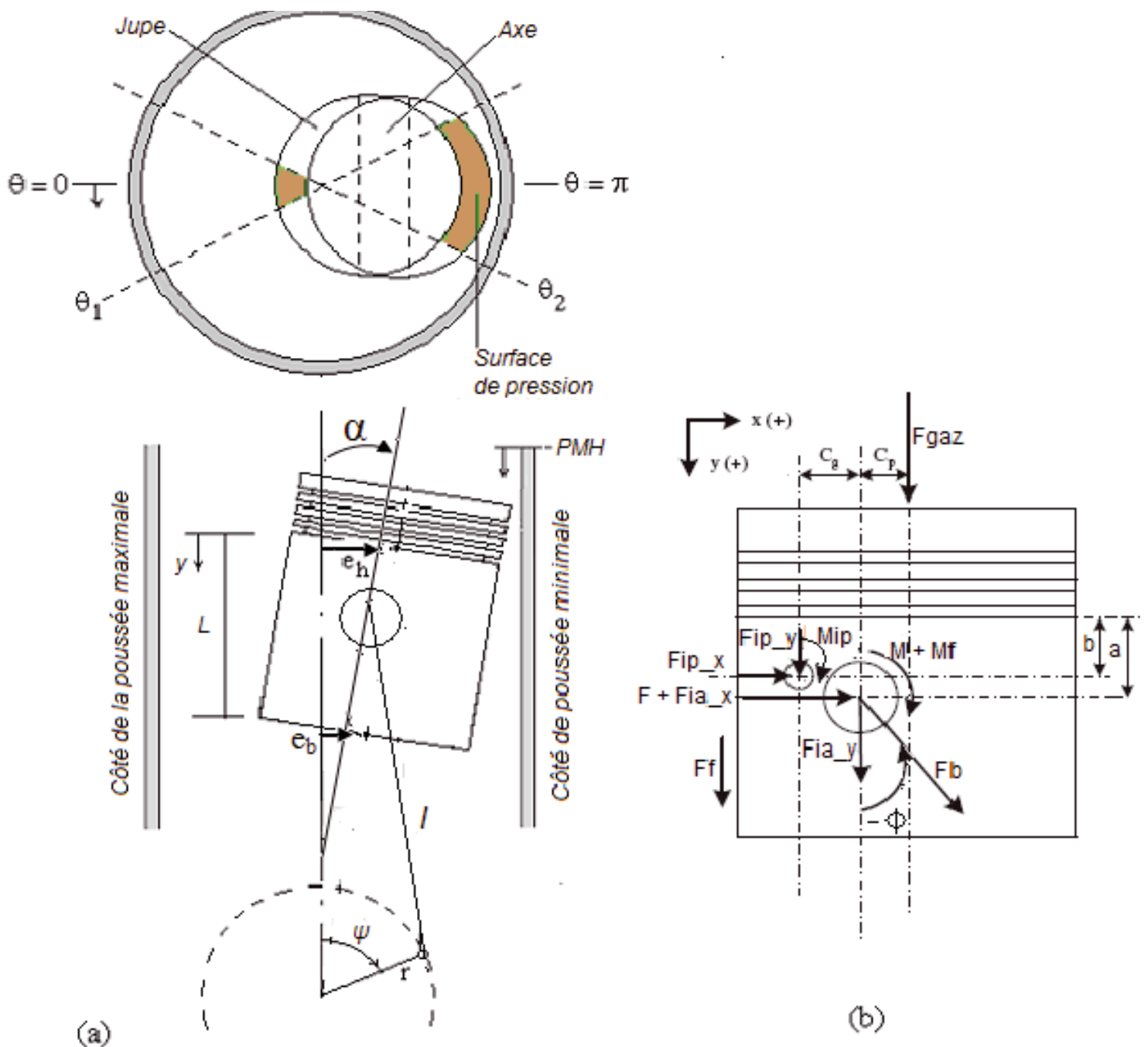

(b)

Fig. 1. (a) Système piston/cylindre, (b) forces et moments agissant sur le piston.

latéral et au mouvement de basculement. Le schéma du système piston/cylindre est représenté dans la figure 1a. Toutes les forces et les moments agissant sur le piston sont indiqués dans la figure $1 \mathrm{~b}$. La position, la vitesse et l'accélération du piston le long de l'axe du cylindre sont en fonction de l'angle du vilebrequin $\psi$. La translation du piston le long de la direction perpendiculaire à l'axe du cylindre et la rotation autour de l'axe du piston sont très petites mais extrêmement importantes. Ces mouvements peuvent être mieux décrits par les excentricités du piston $e_{\mathrm{h}}(t)$ et $e_{\mathrm{b}}(t)$, en haut et en bas de la jupe respectivement.

Les équations d'équilibre des forces et des moments par rapport à l'axe du piston appliqués à l'ensemble piston et axe sont données par les relations suivantes :

$$
\begin{gathered}
F+F_{i \mathrm{a}_{\_} x}+F_{i \mathrm{p} \__{-}}-F_{\mathrm{b}} \sin \phi=0 \\
F_{\mathrm{gaz}}+F_{\mathrm{f}}+F_{i \mathrm{a}_{\mathrm{a} y}}+F_{i \mathrm{p} \_y}+F_{\mathrm{b}} \cos \phi=0
\end{gathered}
$$

$$
M+M_{\mathrm{f}}+M_{i \mathrm{p}}+F_{i \mathrm{p} \_x}(a-b)-F_{i \mathrm{p} \_y} C_{\mathrm{g}}+F_{\mathrm{gaz}} C_{\mathrm{p}}=0
$$

En éliminant $F_{\mathrm{b}}$ des équations (1) et (2), on obtient les équations suivantes :

$$
\begin{aligned}
-F_{i \mathrm{a} \_x}-F_{i \mathrm{p} \_x} & =F_{\mathrm{s}}+F+F_{\mathrm{f}} \tan \phi \\
-M_{i \mathrm{p}}-F_{i \mathrm{p} \_x}(a-b) & =M_{\mathrm{s}}+M+M_{\mathrm{f}}
\end{aligned}
$$

avec

$$
\begin{aligned}
& F_{\mathrm{S}}=\left(F_{\mathrm{gaz}}+F_{i \mathrm{a}_{\_} y}+F_{i \mathrm{p} \_y}\right) \tan \phi \\
& M_{\mathrm{s}}=F_{\mathrm{gaz}} C_{\mathrm{p}}-F_{i_{\mathrm{p}} y} C_{\mathrm{g}} \\
& \phi=\tan ^{-1}\left(B\left(l^{2}-B^{2}\right)^{-0,5}\right) \\
& B=C_{\mathrm{p}}+r \sin \psi \\
& F_{i \mathrm{p} \_y}=-m_{\mathrm{p}} \ddot{Y} \\
& F_{i \mathrm{a} \_y}=-m_{\mathrm{a}} \ddot{Y}
\end{aligned}
$$


Les forces et le moment d'inertie du mouvement secondaire sont donnés par :

$$
\begin{aligned}
F_{i \mathrm{p} \_x} & =-m_{\mathrm{p}}\left(\ddot{e_{\mathrm{h}}}+\frac{b}{L}\left(\ddot{e}_{\mathrm{b}}-\ddot{e}_{\mathrm{h}}\right)\right) \\
F_{i \mathrm{a} \_x} & =-m_{\mathrm{a}}\left(\ddot{e}_{\mathrm{h}}+\frac{a}{L}\left(\ddot{e}_{\mathrm{b}}-\ddot{e}_{\mathrm{h}}\right)\right) \\
M_{i \mathrm{p}} & =-I_{\mathrm{p}} \frac{\left(\ddot{e}_{\mathrm{h}}-\ddot{e}_{\mathrm{b}}\right)}{L}
\end{aligned}
$$

En remplaçant les équations (6), (7) et (8) dans (4) et (5), on obtient les équations de mouvement suivantes :

$$
\begin{array}{r}
{\left[\begin{array}{cc}
m_{\mathrm{p}}\left(1-\frac{b}{L}\right)+m_{\mathrm{a}}\left(1-\frac{a}{L}\right) & m_{\mathrm{p}} \frac{b}{L}+m_{\mathrm{a}} \frac{a}{L} \\
\frac{I_{\mathrm{p}}}{L}+m_{\mathrm{p}}(a-b)\left(1-\frac{b}{L}\right) & m_{\mathrm{p}}(a-b) \frac{b}{L}-\frac{I_{\mathrm{p}}}{L}
\end{array}\right]\left[\begin{array}{c}
\ddot{e}_{\mathrm{h}} \\
\ddot{e}_{\mathrm{b}}
\end{array}\right]} \\
=\left[\begin{array}{c}
F+F_{\mathrm{s}}+F_{\mathrm{f}} \tan \Phi \\
M+M_{\mathrm{s}}+M_{\mathrm{f}} l
\end{array}\right]
\end{array}
$$

La résolution de l'équation (9), permet de déterminer les excentricités $e_{\mathrm{h}}$ et $e_{\mathrm{b}}$. Lorsque $F_{\text {gaz }}$ est donnée, $F_{\mathrm{s}}$ et $M_{\mathrm{s}}$ peuvent facilement être calculés. Le problème qui se pose maintenant est de savoir comment déterminer $F$, $F_{\mathrm{f}}, M$ et $M_{\mathrm{f}}$ avant de résoudre l'équation (9). Toutes ces forces et moments sont considérés comme la somme d'une partie hydrodynamique et une partie de contact solide et peuvent être déterminés selon le modèle discuté ci-dessous.

\section{2 Équation de Reynolds}

L'équation de Reynolds, qui régit la génération de la pression hydrodynamique d'un fluide incompressible à écoulement laminaire est donnée par :

$$
\frac{\partial}{\partial y}\left(\frac{h^{3}}{6 \mu} \frac{\partial p_{h}}{\partial y}\right)+\frac{1}{R^{2}} \frac{\partial}{\partial \theta}\left(\frac{h^{3}}{6 \mu} \frac{\partial p_{h}}{\partial \theta}\right)=U \frac{\partial h}{\partial y}+2 \frac{\partial h}{\partial t}
$$

La détermination du champ de pression dans le film implique la connaissance de la pression aux frontières du domaine de résolution :

- dans la direction axiale : $P(\theta, y)=0$ pour $y=0$ et $y=L($ quel que soit $\theta)$

- dans la direction circonférentielle : pour un profil symétrique et circulaire de la jupe : $\frac{\partial P}{\partial \theta}=0$ pour $\theta=0$ et $\theta=\pi$ en plus $P=0$ pour $\theta_{1} \leq \theta \leq \theta_{2}$, où $\theta_{1}$ et $\theta_{2}$ désignent l'étendue angulaire du lubrifiant sur les côtés de la poussée maximale et la poussée minimale respectivement. Le domaine lubrifié et la géométrie du piston sont représentés dans la figure 2 .

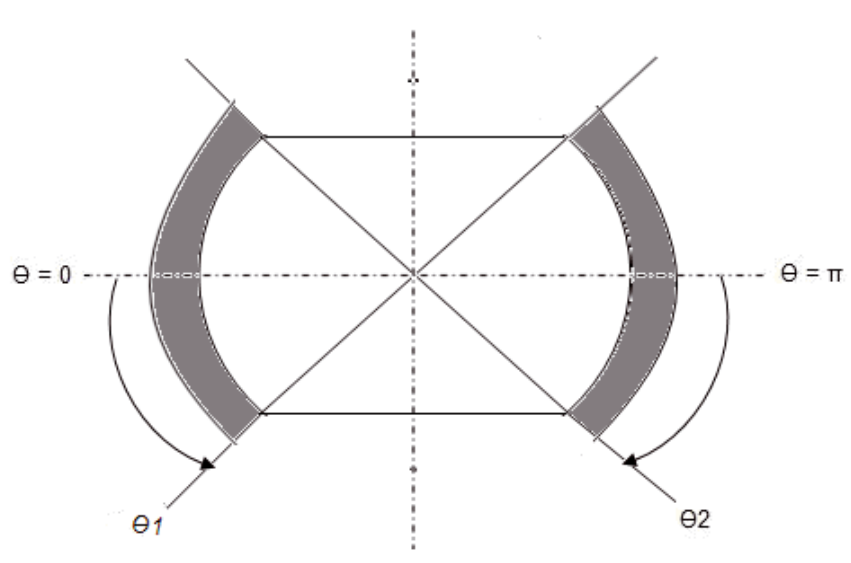

(a)

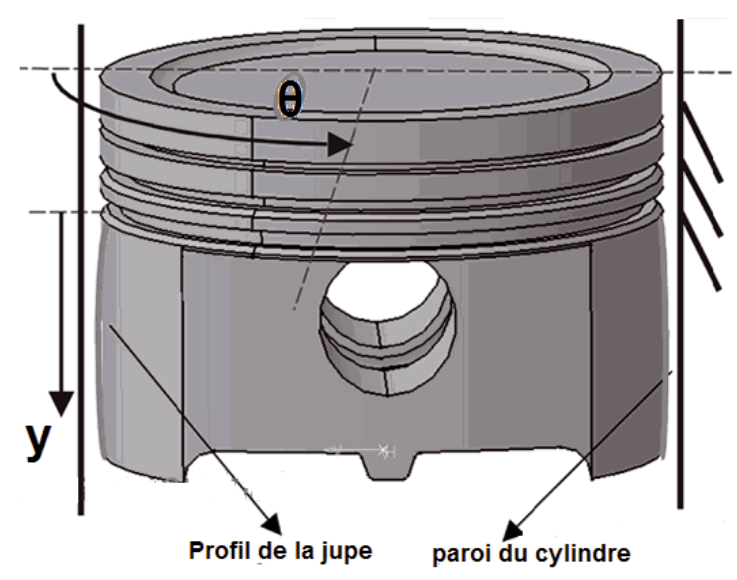

(b)

Fig. 2. (a) Étendue angulaire du lubrifiant, (b) géométrie du piston.

\section{3 Épaisseur du film lubrifiant}

L'épaisseur moyenne du film d'huile entre la jupe et la paroi du cylindre peut être exprimée par

$$
h=e_{\mathrm{h}} \cos \theta+\left(e_{\mathrm{b}}-e_{\mathrm{h}}\right) \frac{y}{L} \cos \theta+q(y)+h_{\mathrm{e}}
$$

où $q(y)$ représente l'épaisseur géométrique nominale du film à la coordonnée $y$ pour un profil axial arbitraire de la jupe, et $h_{\mathrm{e}}$ l'épaisseur due à la déformation élastique de la jupe.

La viscosité est considérée comme une fonction de la température du fluide, son expression est donnée par :

$$
\mu=\mu_{0} e(-\beta \Delta T)
$$

où $\mu_{0}$ est la viscosité d'huile à $T_{0}$ et $\beta$ est le coefficient de thermoviscosité.

\section{Force hydrodynamique}

La pression hydrodynamique est obtenue par la résolution de l'équation de Reynolds. Ainsi les forces et 


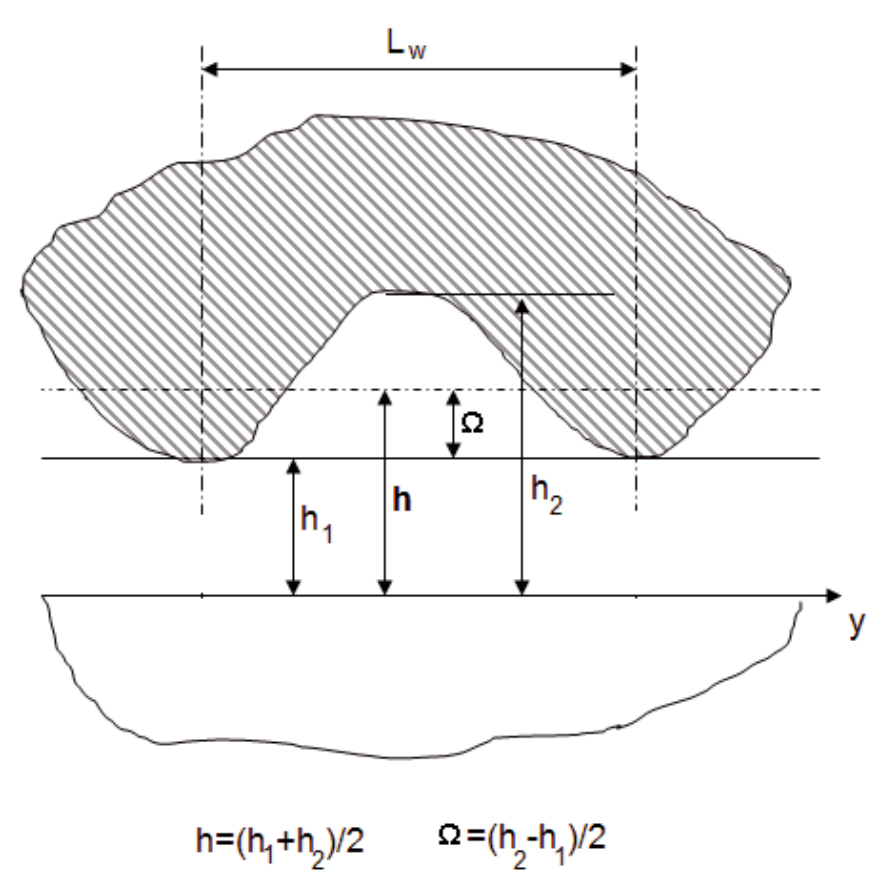

Fig. 3. Modèle de base d'une ondulation.

les moments hydrodynamiques peuvent être calculés par les intégrations suivantes :

$$
\begin{aligned}
F_{\mathrm{h}} & =2 R \int_{0}^{\pi} \int_{0}^{L} P_{\mathrm{h}} \cos \theta \mathrm{d} \theta \mathrm{d} y \\
M_{\mathrm{h}} & =2 R \int_{0}^{\pi} \int_{0}^{L} P_{\mathrm{h}}(a-y) \cos \theta \mathrm{d} \theta \mathrm{d} y \\
F_{\mathrm{fh}} & =2 R \int_{0}^{\pi} \int_{0}^{L} \tau \mathrm{d} \theta \mathrm{d} y \\
M_{\mathrm{fh}} & =2 R \int_{0}^{\pi} \int_{0}^{L} \tau\left(R \cos \theta-C_{\mathrm{p}}\right) \mathrm{d} \theta \mathrm{d} y
\end{aligned}
$$

avec

$$
\tau=-\frac{\mu U}{h}+\frac{h}{2} \frac{\partial P_{\mathrm{h}}}{\partial y}
$$

$\tau$ est la contrainte de cisaillement agissant sur la surface de la jupe du piston.

\section{Force de contact}

La solution pour la pression de contact solidesolide, $P_{\mathrm{c}}$, est adaptée des références $[1,12]$. Le modèle géométrique des ondulations de surface est présenté dans la figure 3. En utilisant une notation similaire, la pression de contact $P_{\mathrm{c}}$ peut être résolue en commençant par la corrélation suivante :

$$
\delta=x_{i}\left[-0,635 \ln \left(\frac{x_{i} L_{\mathrm{w}}}{4 \Omega}\right)+1,0556\right]
$$

où $\Omega, L_{\mathrm{w}}$ sont l'amplitude et la longueur de l'ondulation respectivement. La déformée imposée par le contact solide-solide dans le plateau de l'ondulation, $\delta$, est donnée par :

$$
\delta=\Omega-h, \text { si } \Omega\rangle h, \text { et } \delta=0 \text { autrement }
$$

Ainsi, à tout moment $t, \delta$ est connu si $h$ est connu, et la quantité $x_{i}$ peut être déterminée à partir de l'équation (18). La pression de contact $P_{\mathrm{c}}$ est donnée par :

$$
P_{\mathrm{c}}=x_{i} \frac{E^{\prime}}{L_{\mathrm{w}}}
$$

La pression de contact ainsi évaluée ci-dessus est en accord avec la solution approchée donnée par Zhu [2], pour un piston en aluminium :

$$
P_{\mathrm{c}}=5,464 \times 10^{13} \delta^{1,0552}
$$

Les forces et les moments de contact peuvent être calculés par :

$$
\begin{aligned}
F_{\mathrm{c}} & =2 R \int_{0}^{\pi} \int_{0}^{L} P_{\mathrm{c}} \cos \theta \mathrm{d} \theta \mathrm{d} y \\
M_{\mathrm{c}} & =2 R \int_{0}^{\pi} \int_{0}^{L} P_{\mathrm{c}}(a-y) \cos \theta \mathrm{d} \theta \mathrm{d} y \\
F_{\mathrm{fc}} & =-2 \mu_{\mathrm{f}} R \frac{|U|}{U} \int_{0}^{\pi} \int_{0}^{L} P_{\mathrm{c}} \mathrm{d} \theta \mathrm{d} y \\
M_{\mathrm{fc}} & =2 \mu_{\mathrm{f}} R \frac{|U|}{U} \int_{0}^{\pi} \int_{0}^{L} P_{\mathrm{c}}\left(R \cos \theta-C_{\mathrm{p}}\right) \mathrm{d} \theta \mathrm{d} y
\end{aligned}
$$

où $\mu_{\mathrm{f}}$ est le coefficient de frottement, qui est déterminé expérimentalement [1].

D'autre part, la puissance dissipée instantanée $P_{\mathrm{u}}$ est définie par

$$
P_{\mathrm{u}}=F_{\mathrm{f}} U+M_{\mathrm{f}} \dot{\alpha}
$$

où $F_{\mathrm{f}}=F_{\mathrm{fh}}+F_{\mathrm{fc}}$ et $M_{\mathrm{f}}=M_{\mathrm{fh}}+M_{\mathrm{fc}}$

\section{Déformation élastique}

Pour pouvoir calculer les déformations élastiques issues du champ de pression dans le film et de la pression de contact, la technique des matrices de compliance est utilisée. Considérant la tête du piston comme encastrée, donc indéformable, le déplacement radial d'un point situé sur la surface de la jupe dépend de toutes les forces qui agissent sur tous les autres points de cette même surface. En utilisant la méthode des Éléments-Finis (MEF) une matrice de compliance $[C]$ est calculée pour déterminer le déplacement radial des nœuds du maillage sur la surface 
de la jupe. L'expression de l'épaisseur du film en un nœud $i$ est donnée par l'équation suivante :

$$
h_{\mathrm{e}}^{i}=\sum_{k=1}^{n} C(i, k) F_{k}
$$

où $C(i, k)$ est la matrice de compliance élastique qui représente le déplacement au nœud $i$ dû à une force unitaire au nœud $k$. Pour son obtention, une charge unitaire est appliquée successivement en chacun des nœuds $n$ de la structure appartenant à la paroi du film et les déplacements normaux en chaque nœud de cette paroi sont calculés.

$F_{k}$ est la force exercée au nœud $k$ du film lubrifiant résultant du champ de pression et de la pression de contact autour de ce nœud.

Dans la présente étude, le film est maillé en éléments isoparamétriques à huit nœuds, le maillage de la surface du piston qui délimite le film est constitué d'éléments triangulaires à 3 nœuds. La correspondance entre les deux maillages est donnée avec détail dans la référence [21]. Avec 588 nœuds pour le piston complet, on a un compromis entre l'exactitude des solutions et le temps de calcul, l'utilisation de mailles plus petites conduit aux mêmes résultats.

\section{Méthode de résolution}

La résolution des équations générales d'un problème TEHD global nécessite l'utilisation des boucles itératives pour :

- la résolution du problème hydrodynamique afin de trouver la solution $\left(P_{\mathrm{h}}, e_{\mathrm{h}}, e_{\mathrm{b}}\right)$. L'itération se termine lorsque les critères de convergence suivants sont satisfaits :

$$
\begin{gathered}
\left|\frac{P_{\mathrm{h}, i j}^{k}-P_{\mathrm{h}, i j}^{k-1}}{P_{\mathrm{h}, i j}^{k}}\right|\left\langle 10^{-4}\right. \\
\left|\frac{e_{\mathrm{h}}^{k}-e_{\mathrm{h}}^{k-1}}{e_{\mathrm{h}}^{k}}\right|<10^{-3} \\
\left|\frac{e_{\mathrm{b}}^{k}-e_{\mathrm{b}}^{k-1}}{e_{\mathrm{b}}^{k}}\right|<10^{-3}
\end{gathered}
$$

- la détermination de toutes les forces et les moments hydrodynamiques et de contacts qui agissent sur le piston;

- la résolution du problème élastique : trouver la déformée de la jupe créée par la pression totale ;

- la détermination de la température et de la viscosité par une approche thermique globale.

Le schéma numérique de la figure 4 résume cette démarche.

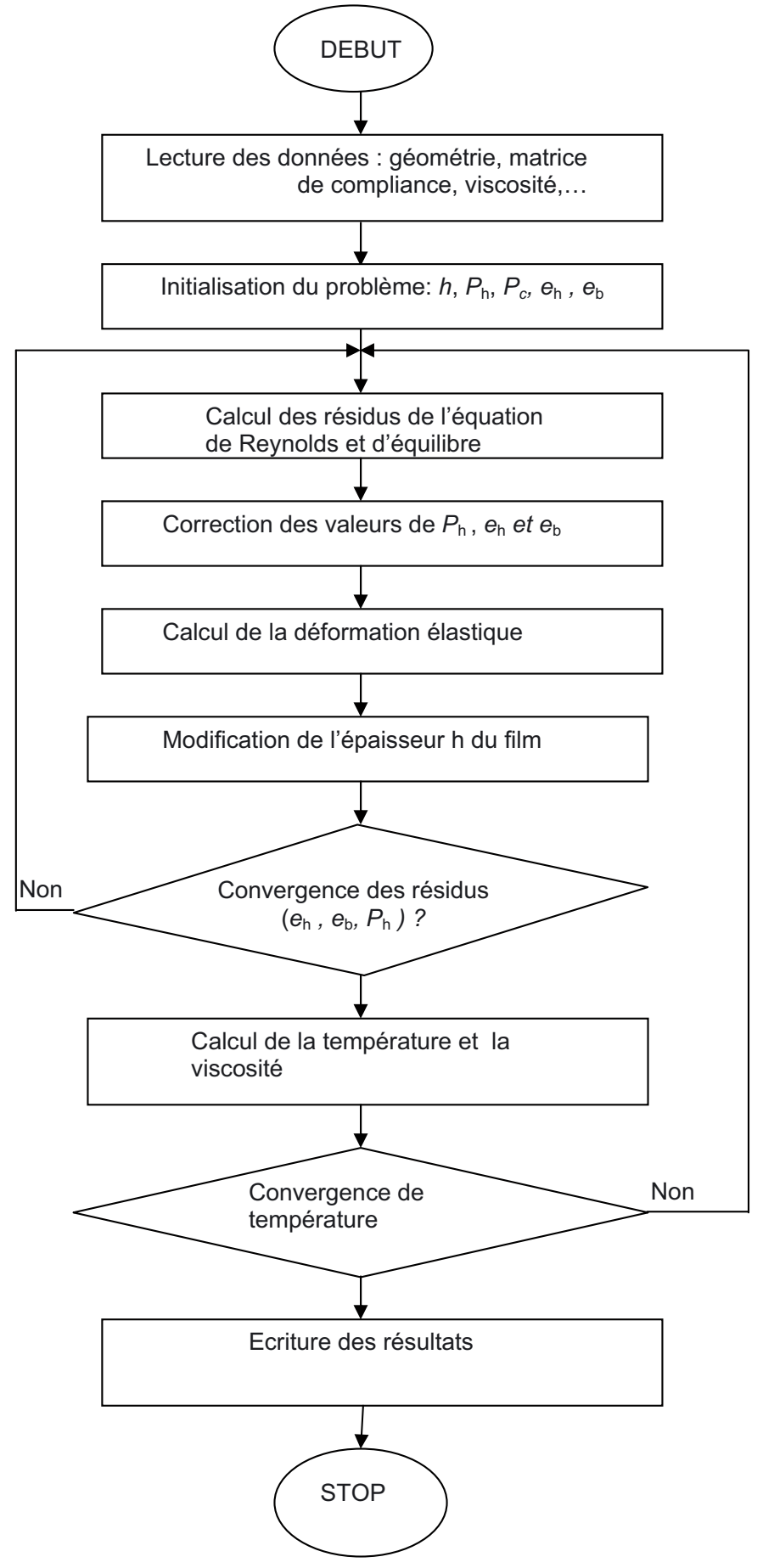

Fig. 4. Organigramme général.

\section{Résultats et discussion}

Le modèle est étudié avec une série de paramètres d'entrée initiale, énumérés dans le tableau 1. Le diagramme de la force des gaz de combustion basée sur la pression donnée pour chaque pas de temps est tracé dans la figure 5 en fonction de l'angle du vilebrequin. 
Tableau 1. Paramètres d'entrée.

\begin{tabular}{lc}
\hline Paramètres & Valeurs \\
\hline Diamètre du piston $(\mathrm{mm})$ & 83 \\
Rayon du vilebrequin $(\mathrm{mm})$ & 41,8 \\
Longueur de bielle $(\mathrm{mm})$ & 133 \\
Longueur de la jupe du piston (mm) & 33,8 \\
Angle fluide (degrés) & 37,5 \\
Jeu radial ( $\mu \mathrm{m})$ & 10 \\
Désaxage piston-axe (mm) & 1 \\
Désaxage centre de gravité (mm) & 2 \\
Distance haut jupe-axe (mm) & 12,5 \\
Distance haut jupe-centre de gravité (mm) & 1,5 \\
Masse de l'axe (kg) & 0,09 \\
Masse du piston (kg) & 0,295 \\
Module d'élasticité de Young de la jupe (GPa) & 200 \\
Viscosité dynamique du lubrifiant à $40{ }^{\circ} \mathrm{C}(\mathrm{Pa} . \mathrm{s})$ & 0,0192 \\
Amplitude des ondulations $(\mu \mathrm{m})$ & 3 \\
Vitesse de rotation (tr.min $\left.{ }^{-1}\right)$ & 1000 \\
Coefficient du frottement de contact & 0,150 \\
\hline
\end{tabular}

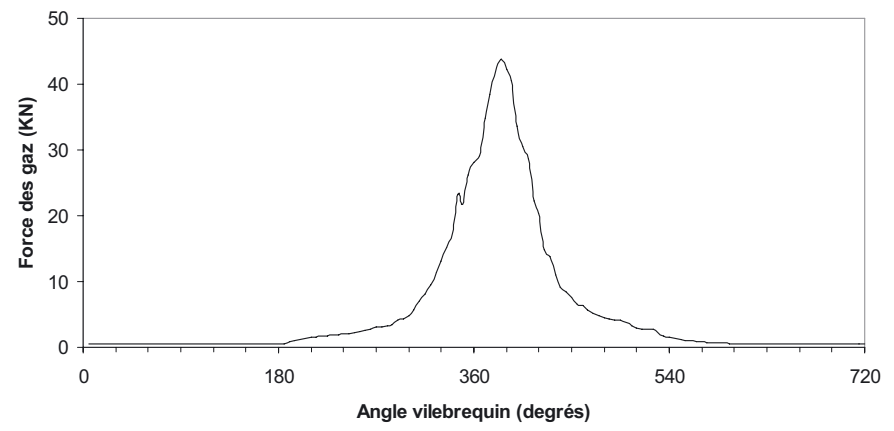

Fig. 5. Variation de la force des gaz de combustion.

\subsection{Influence du comportement élastique}

Les résultats de la simulation du frottement total sont reportés dans la figure 6 . La courbe de frottement est la composante de deux parties, un frottement hydrodynamique repéré par $F_{\mathrm{h}}$ et un frottement dû à un contact solide-solide repéré par $F_{\mathrm{c}}$. Pour une jupe rigide, on remarque un niveau élevé de frottement au cours de la course de détente, lorsque la pression des gaz de combustion est élevée. À ces instants, l'action hydrodynamique est faible et les contacts solide-solide sont dominants. Quand un point de la surface de la jupe arrive à proximité de l'alésage, des hautes pressions déforment la surface, afin d'augmenter la séparation entre les deux surfaces. Ce mécanisme étant absent avec une jupe rigide, elle peut facilement entrer en contact avec les limites de l'alésage, comparativement à une jupe élastique.

La figure 7 montre la puissance dissipée instantanée en fonction de l'angle du vilebrequin, cette puissance est proportionnelle à la vitesse axiale du piston (Fig. 8), ce qui explique l'allure sinusoïdale de cette courbe. Les grands pics observés pour une jupe rigide au cours de la phase combustion-détente sont causés par le gradient de

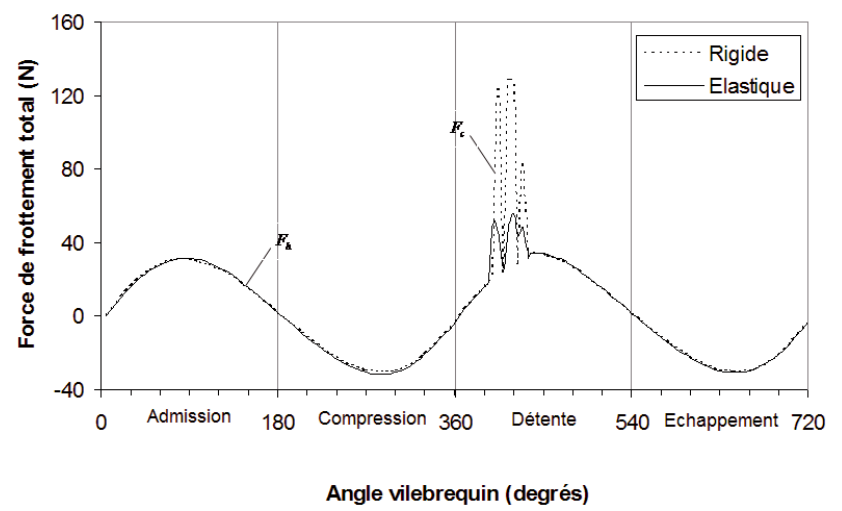

Fig. 6. Force de frottement totale (comparaison élastiquerigide).

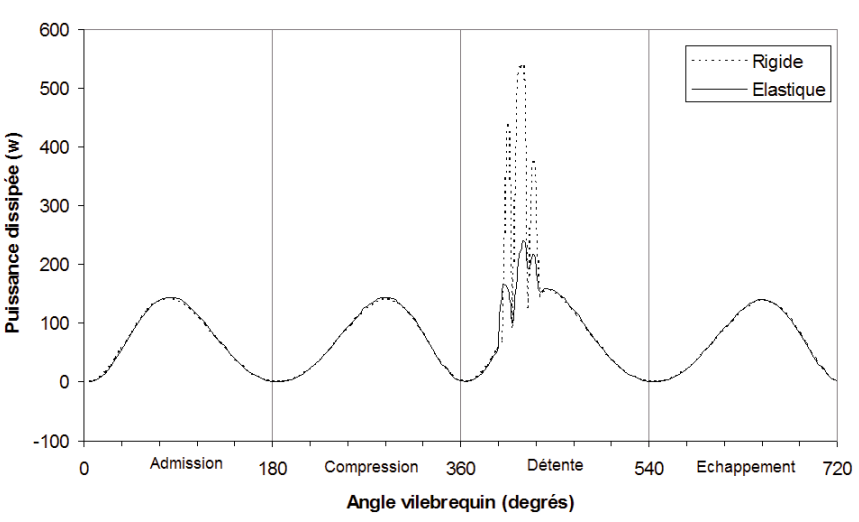

Fig. 7. Puissance dissipée (comparaison élastique-rigide).

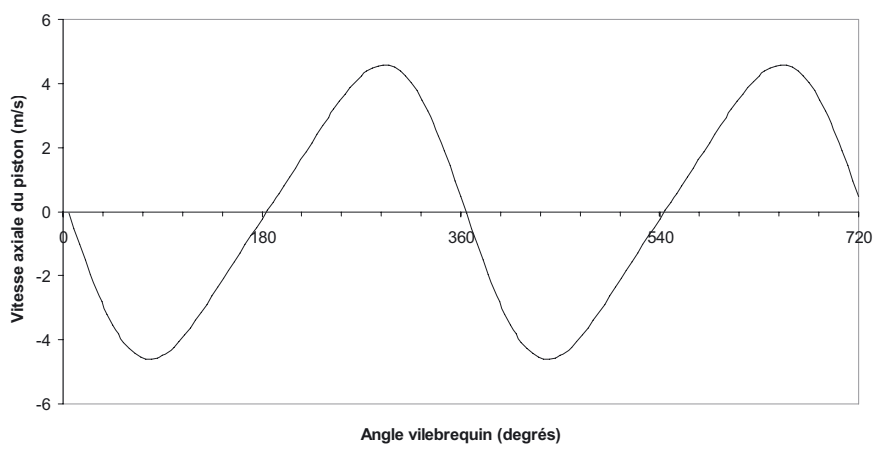

Fig. 8. Vitesse axiale du piston.

pression dont dépend la force de frottement (Éqs. (15) et (17)) d'une part, et par les contacts solides sévères au cours de cette phase d'autre part. Pour une jupe élastique, la déformation est uniforme sur une grande zone, et le piston est de plus en plus soumis aux forces hydrodynamiques (et moins aux forces de contact solide-solide). Ces résultats sont reportés sur la figure 9 qui présente une comparaison de la distribution des pressions hydrodynamique et de contact entre une jupe rigide et une jupe élastique. La perte de puissance totale due à la force de contact diminue dans la mesure où la flexibilité de la jupe 

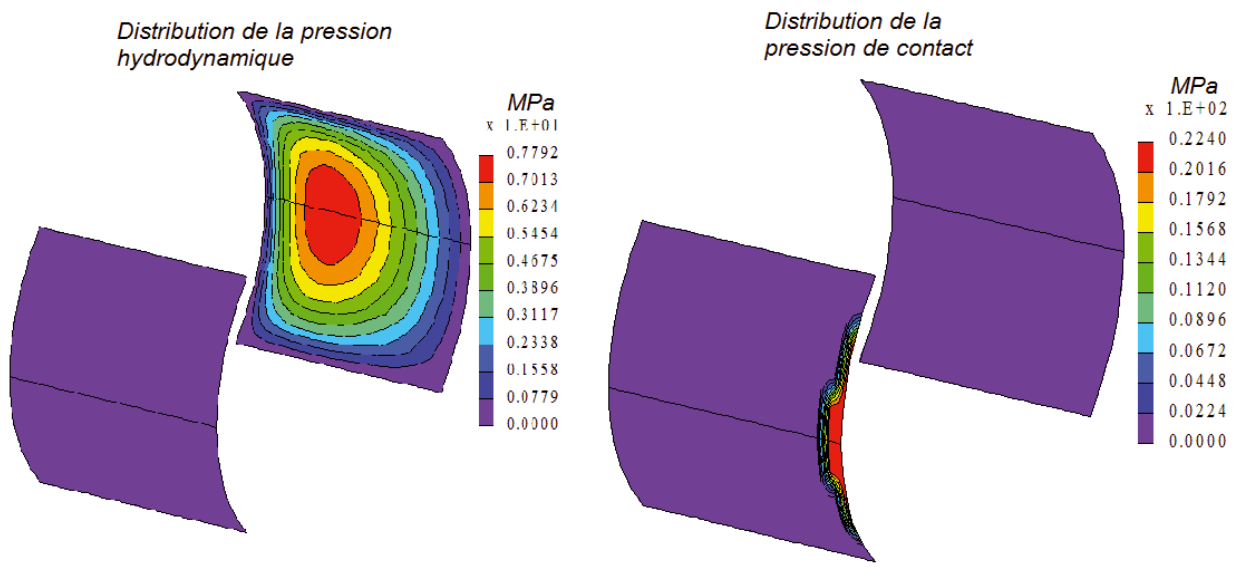

a) jupe rigide
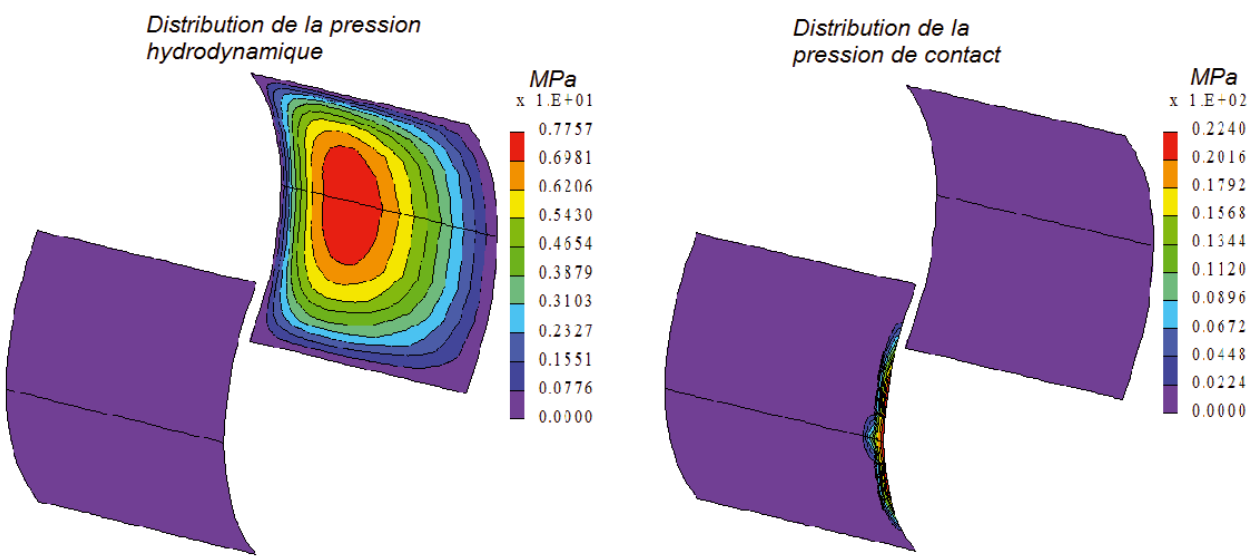

b) jupe élastique

Fig. 9. Distribution des pressions hydrodynamiques et de contact à l'angle de vilebrequin $420^{\circ}$ pour (a) jupe rigide (b) jupe élastique.

augmente (baisse des forces de frottement de contact), comme le montrent les figures 6 et 7 .

Le tableau 2 présente les résultats obtenus avec un modèle rigide et un autre élastique. On constate que l'épaisseur minimale du film dans le cas élastique est nettement plus élevée que dans le cas rigide. L'augmentation est d'environ $36 \%$. Il en résulte une légère diminution de la pression hydrodynamique maximale. Une nette régression du contact solide-solide est remarquée lorsque on passe du rigide à l'élastique, cette régression est observée tant en étendue qu'en valeur maximale, la diminution est de $32 \%$. La diminution du contact solide affecte directement la force de frottement totale, puisque cette dernière diminue presque de $42 \%$ en moyenne sur un cycle. La puissance dissipée et la force de frottement totale sont nettement plus grandes dans le cas rigide qu'en élastique.

\subsection{Influence du profil pour une jupe rigide}

Pour étudier l'influence du profil axial de la jupe sur les caractéristiques tribologiques du piston, on a choisi
Tableau 2. Comparaison entre modèle rigide et élastique.

\begin{tabular}{lcc}
\hline Caractéristiques & \multicolumn{2}{c}{ Cas rigide Cas élastique } \\
\hline Épaisseur minimale du film & $2,2 \mu \mathrm{m}$ & $3,0 \mu \mathrm{m}$ \\
Pression hydrodynamique maximale & $12,8 \mathrm{MPa}$ & $12,3 \mathrm{MPa}$ \\
Pression de contact maximale & $22,4 \mathrm{MPa}$ & $15,2 \mathrm{MPa}$ \\
Force de frottement totale moyenne & $22,2 \mathrm{~N}$ & $20,7 \mathrm{~N}$ \\
Puissance dissipée moyenne & $78,5 \mathrm{~W}$ & $73,5 \mathrm{~W}$ \\
\hline
\end{tabular}

deux profils pour une jupe rigide : un profil plat et un profil bombé. La figure 10 montre les deux profils de la jupe considérés.

En comparant les résultats des deux profils, il est observé que, pour un profil plat, la force de frottement et la puissance dissipée sont les plus faibles (Figs. 11 et 12). Un profil plat de la jupe du piston diminue la tendance du contact solide-solide et donc réduit le frottement total de la jupe du piston. Avec un profil bombé le soutien hydrodynamique est faible et un frottement limite s'installe, conduisant à une augmentation de la puissance dissipée par frottement. En plus, la surface inondée d'un profil bombé est réduite, et la pression est en moyenne plus 


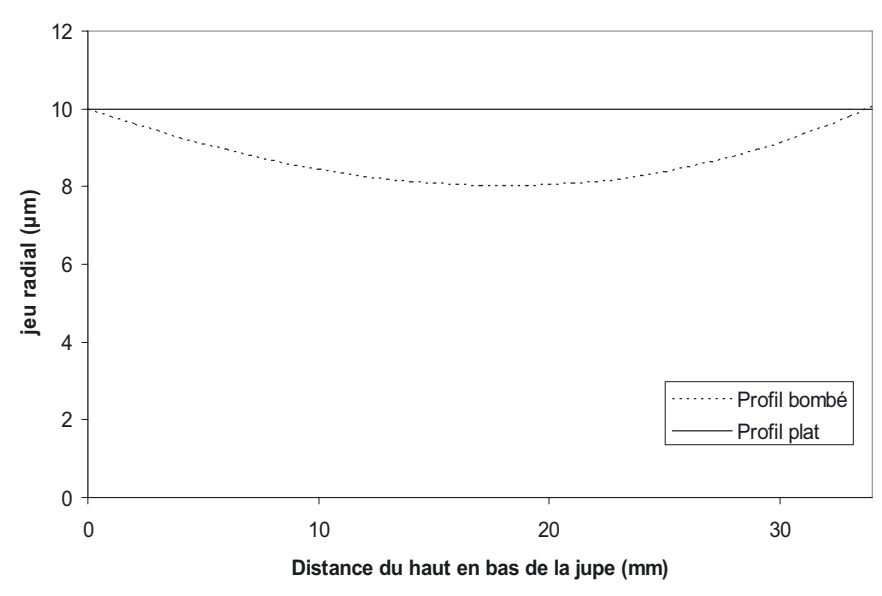

Fig. 10. Profils de la jupe.

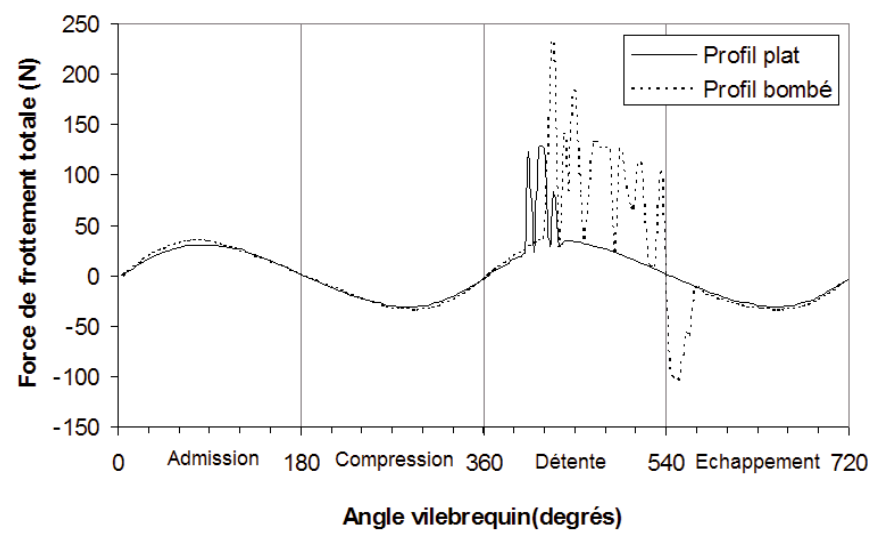

Fig. 11. Effet du profil de la jupe sur la force de frottement totale.

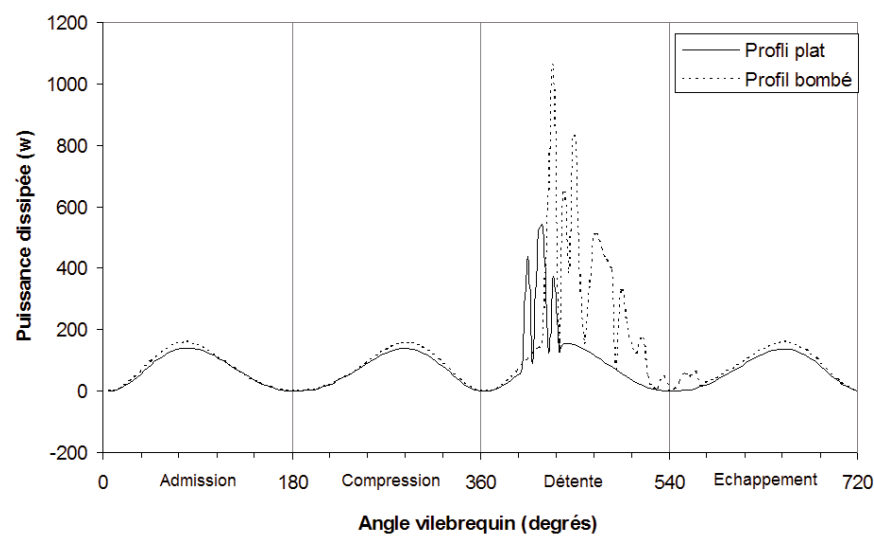

Fig. 12. Effet du profil de la jupe sur la puissance dissipée.

élevée par rapport à un profil plat. Le gradient de pression est réduit pour un profil plat, le piston est plus susceptible de rester dans un régime de lubrification hydrodynamique, par contre, on observe, pour un profil bombé, des pics de pression élevée qui conduit à des contacts limites (Fig. 13). Le phénomène de frottement et de dissipation est observé au cours de la course combustion-détente pour les deux profils, pendant cette course, la pression dans le mécanisme est à son maximum.
Tableau 3. Comparaison entre profil plat et bombé.

\begin{tabular}{lcc}
\hline Caractéristiques & \multicolumn{2}{c}{ Profil plat Profil bombé } \\
\hline Épaisseur minimale du film & $2,2 \mu \mathrm{m}$ & $2,8 \mu \mathrm{m}$ \\
Pression hydrodynamique maximale & $12,8 \mathrm{MPa}$ & $13,0 \mathrm{MPa}$ \\
Pression de contact maximale & $22,4 \mathrm{MPa}$ & $26,1 \mathrm{MPa}$ \\
Force de frottement totale moyenne & $22,2 \mathrm{~N}$ & $35,6 \mathrm{~N}$ \\
Puissance dissipée moyenne & $78,5 \mathrm{~W}$ & $115,0 \mathrm{~W}$ \\
\hline
\end{tabular}

Dans le tableau 3, on trouve les valeurs maximales des pressions et l'épaisseur minimale du film d'huile avec un profil plat et un autre bombé. Le minimum de l'épaisseur est plus grand pour le profil bombé, cette augmentation est de $20 \%$ juste après la combustion, par contre sur l'ensemble du cycle, on constate une élévation globale de l'épaisseur minimale dans le cas du profil bombé (Fig. 14). Le maximum de la pression hydrodynamique ne varie que légèrement, mais sur l'ensemble du cycle, on constate l'apparition de zone de pression plus élevée dans le cas du profil bombé (Fig. 15). Ces élévations sont liées au rétrécissement de la zone lubrifiée comme celle mentionnée sur la figure 13. Cette diminution de la zone active entraîne plus de contact solide-solide, et donc une augmentation de la force de frottement et de la puissance dissipée. Le potentiel de réduction de la puissance dissipée par frottement en changeant le profil de la jupe est d'environ $30 \%$.

\section{Conclusion}

Une analyse numérique du mouvement du piston a été effectuée afin d'étudier l'effet de l'élasticité et du profil de la jupe sur le comportement tribologique du piston. Les observations suivantes résument les résultats.

1. Une jupe élastique permet de réduire le frottement et la puissance dissipée par rapport à une jupe rigide. Les forces hydrodynamiques et les forces de contact déforment plus facilement une jupe élastique, la surface lubrifiée augmente et la distribution de la pression à l'interface jupe-chemise devient plus uniforme. La réduction du frottement provient principalement de la réduction du frottement des contacts limites.

2. Un profil plat du piston diminue la perte de puissance par frottement total de la jupe. Sur ce profil, la surface lubrifiée et les forces latérales deviennent plus grandes. La perte de puissance par frottement de contact augmente beaucoup plus dans le cas d'un profil bombé.

3 . Près de $60 \%$ de la perte de puissance par frottement de la jupe durant le cycle du moteur provient au cours de la course motrice. Bien que les pertes hydrodynamiques se produisent régulièrement au cours des quatre courses, le frottement de contact limite se produit exclusivement durant la course motrice. Sur le cycle moteur, le rapport du frottement limite au frottement hydrodynamique est de $6 / 4$. 

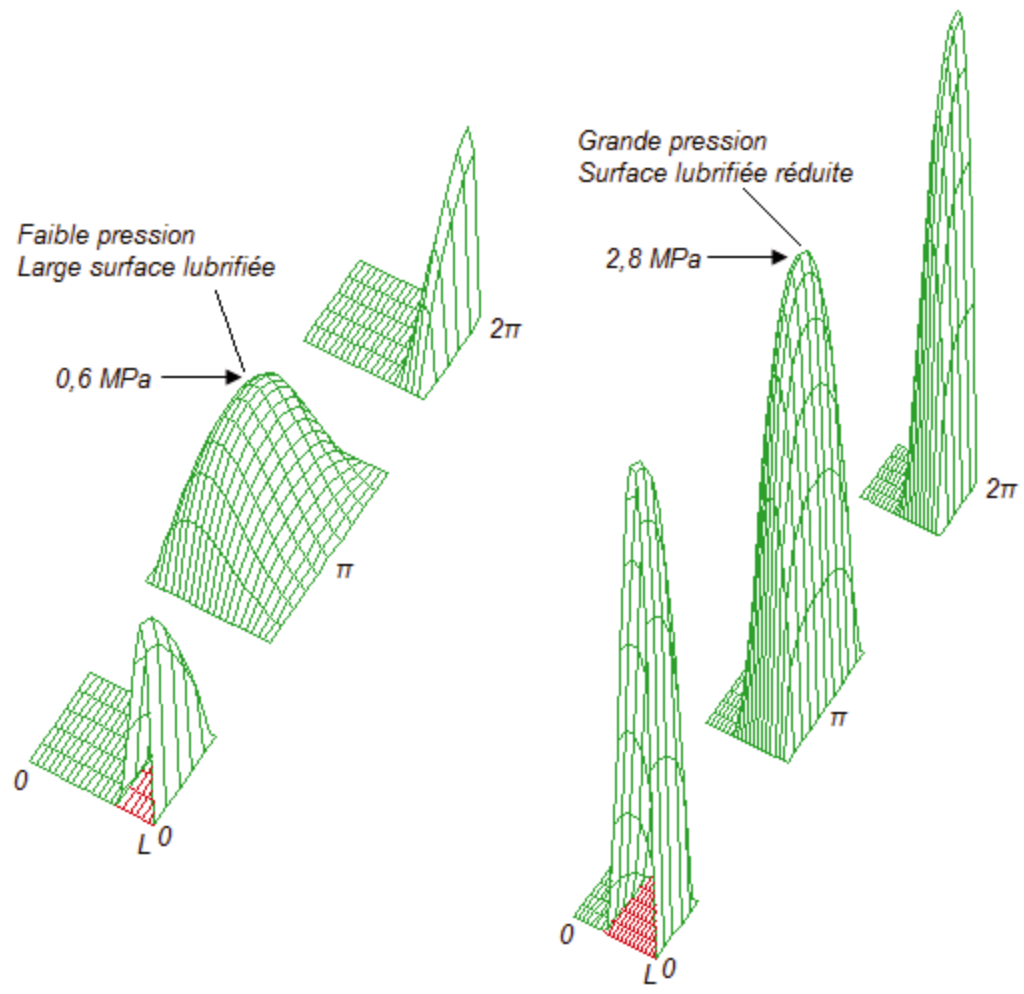

a) Profil plat

b) Profil bombé

Fig. 13. Comparaison du champ de pression hydrodynamique pour profil plat (a) et bombé (b) à l'angle de vilebrequin $630^{\circ}$.

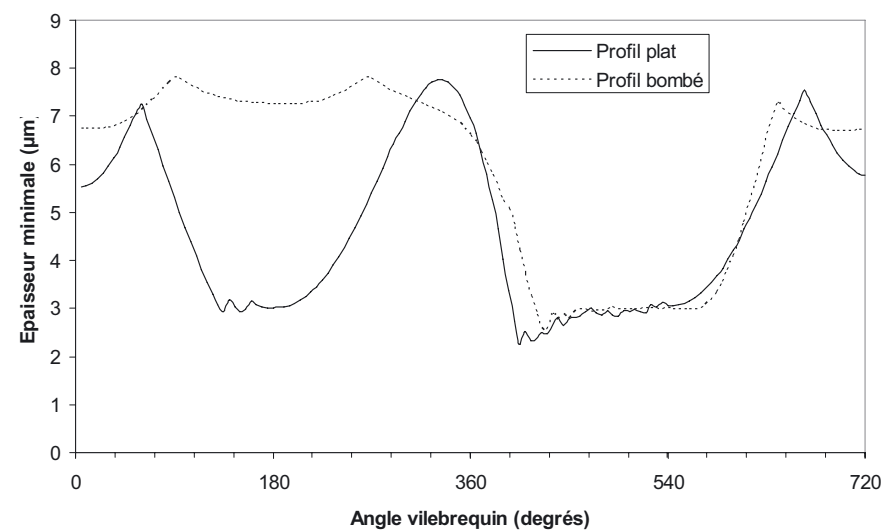

Fig. 14. Épaisseur minimale du film d'huile.

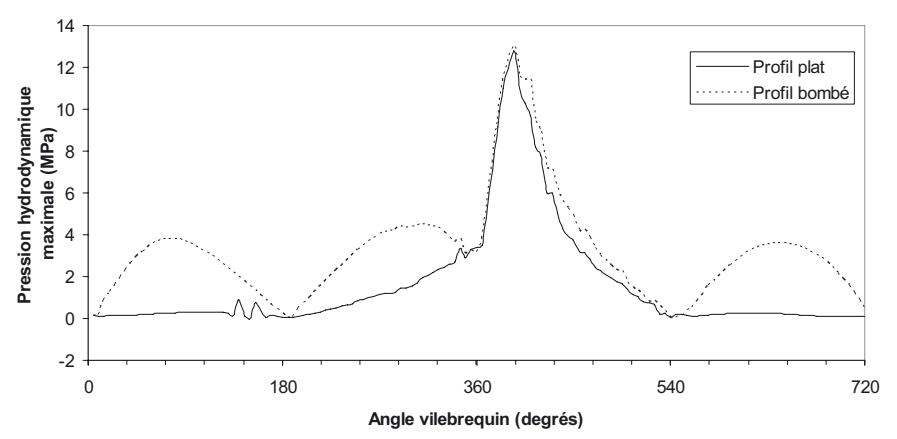

Fig. 15. Pression hydrodynamique maximale.

\section{Références}

[1] D. Zhu, H.S. Cheng, A numerical analysis for piston skirt in mixed lubrication, Part I: Basic modelling, ASME J. Tribology 114 (1992) 553-562

[2] D. Zhu, H.S. Cheng, A numerical analysis for piston skirt in mixed lubrication, Part II: Deformation considerations, ASME J. Tribology 115 (1993) 125-133

[3] R. Keribar, Z. Dursunkaya, A comprehensive model of piston skirt lubrication, SAE Paper 920483, 1992

[4] Z. Dursunkaya, R. Keribar, V. Ganapathy, A model of piston secondary motion and elastohydrodynamic skirt lubrication, ASME Paper 93-ICE-7, Energy-Sources Technology Conference \& Exhibition, Houston, 1993

[5] R. Keribar, Z. Dursunkaya, V. Ganapathy, An integrated design analysis methodology to address piston tribological issues, SAE Paper 930793, 1993

[6] G.D. Knoll, H.J. Peeken, Hydrodynamic lubrication of piston skirt, ASME J. Tribology 104 (1982) 504-509

[7] D.F. Li, S.M. Rohde, H.A. Ezzat, An automotive piston lubrication model, ASLE Trans. 26 (1982) 151-160

[8] T. Suzuki, Y. Fujimoto, Y. Ochiai, I. Fujimura, A numerical study on piston slap in diesel engines, JSME Transactions Series B 53 (1987) 2610-2618

[9] C.H. Li, Thermoelastic behavior of an aluminium diesel engine piston, SAE Paper 860136, 1986

[10] W.B. Blair, D.P. Hoult, V.W. Wong, The role of piston distortion on lubrication in a reciprocating engine, ASME Paper, No. 90-ICE-21, ASME Energy Sources Technology Conference, New Orleans, 1990 
[11] M. Okubo, H. Kanda, T. Yonezawa, Analysis and reduction of piston slap noise in diesel engines, SAE Paper 890127, 1989

[12] V.W. Wong, T. Tian, H. Lang, P. Ryan, Y. Sekia, Y. Kobayashi, S. Aoyama, A numerical model of piston secondary motion and piston slap in partially flooded elastohydrodynamic skirt lubrication, Annual Congress and Exposition, Detroit, MI, SAE Paper 940696, 1994

[13] J.E. Forbes, E.S. Taylor, A method for studying piston friction, NACA Wartime Report, W-7, 1943

[14] S. Furuhama, M. Takiguchi, Measurement of piston friction force in actual operating diesel engine, SAE Paper 790855,1979

[15] S. Furuhama, M. Takiguchi, K. Tomizawa, Effect of piston and piston ring designs on the piston friction forces in diesel engine, SAE Paper 810977, 1981

[16] S. Furuhama, S. Shimichi, New device for the measurement of piston frictional forces in small engines, SAE Sp. Pub. 552, 1983
[17] S. Jang, J. Cho, Effects of skirt profiles on the piston secondary movements by the lubrication behaviours, Int. J. Automotive Techn. 5 (2004) 23-31

[18] S. Balakrishnan, H. Rahnejat, Isothermal transient analysis of piston skirt-to- cylinder wall contacts under combined axial, Lateral and Tilting Motion, J. Phys. D: Appl. Phys. 38 (2005) 787-799

[19] S.H. Mansouri, V.W. Wong, Effects of piston design parameters on piston secondary motion and skirt-liner friction, J. Engineering Tribology, Part J 219 (2005) 435-449

[20] G.A. Livanos, N.P. Kyrtatos, Friction model of a marine diesel engine piston assembly, Tribology Int. 40 (2007) 1441-1453

[21] D. Bonneau, M. Hajjam, Modélisation de la rupture et de la reformation des films lubrifiants dans les contacts elastohydrodynamiques, Rev. Euro. Éléments Finis 10 (2001) 679-704 